\title{
ON THE DERIVED QUOTIENT MODULE $\left({ }^{1}\right)$
}

\author{
BY \\ C. N. WINTON
}

\begin{abstract}
With every $R$-module $M$ associate the direct limit of $\operatorname{Hom}_{R}(D, M)$ over the dense right ideals of $R$, the derived quotient module $\mathscr{D}(M)$ of $M . \mathscr{D}(M)$ is a module over the complete ring of right quotients of $R$. Relationships between $\mathscr{D}(M)$ and the torsion theory of Gentile-Jans are explored and functorial properties of $\mathscr{D}$ are discussed. When $M$ is torsion free, results are given concerning rational closure, rational completion, and injectivity.
\end{abstract}

1. Introduction. Let $R$ be a ring with unity and let $Q$ be its complete ring of right quotients. Following Lambek [8], a right ideal $D$ of $R$ is called dense provided that if an $R$-map $f: E\left(R_{R}\right) \rightarrow E\left(R_{R}\right)$, where $E\left(R_{R}\right)$ denotes the injective hull of $R_{R}$, is such that $f(D)=0$, then $f=0$. It is easy to see this is equivalent to $x D \neq(0)$ for every $x \in E\left(R_{R}\right)$. The collection of dense right ideals is closed under finite intersection; furthermore, any right ideal containing a dense right ideal is again dense. Hence, the collection becomes a directed set if we let $D_{1} \leqq D_{2}$ whenever $D_{2} \subseteq D_{1}$ for dense right ideals $D_{1}$ and $D_{2}$ of $R$. It follows that for any $R$-module $M$ we can form the direct limit $\mathscr{D}(M)$ of $\left\{\operatorname{Hom}_{R}(D, M) \mid D\right.$ is a dense right ideal of $\left.R\right\}$ over the directed set of dense right ideals of $R$. We will call $\mathscr{D}(M)$ the derived quotient module of $M$. It is well known that $\mathscr{D}\left(R_{R}\right)$ has a natural ring structure under which it is naturally isomorphic to $Q . \mathscr{D}(M)$ is a $Q$-module in a natural way.

In this paper the relationship between the derived quotient module and the torsion theory of Gentile-Jans [5], [6] is explored. The functor from $R$-modules to $Q$-modules given by $\mathscr{D}(M)$ is related to extension of scalars and is shown to commute with arbitrary products if and only if $R$ contains a minimal dense right ideal. In particular, perfect rings are shown to have minimal dense right ideals. Finally, when $M$ is torsion free and faithful over $R, \mathscr{D}(M)$ agrees with the rational closure and rational completion of $M$. Further, torsion free $Q$-modules are injective over $R$ if and only if they are injective over $Q$.

2. If $A$ and $B$ are $R$-modules with $A \subseteq B$, then for $b \in B, b^{-1} A$ will always denote $\{x \in R \mid b x \in A\}$. In particular, for $q \in Q$, and $D$ a dense right ideal of $R, q^{-1} D$ is again dense.

Presented to the Society, January 23, 1970; received by the editors February 23, 1970.

AMS 1969 subject classifications. Primary 1640, 1690.

Key words and phrases. Derived quotient module, complete ring of right quotients, dense right ideal, left perfect ring, torsion free module, rational closure, rational completion, torsion submodule, minimal dense right ideal.

( $\left.{ }^{1}\right)$ This paper is based on a portion of the author's doctoral dissertation written at the University of North Carolina at Chapel Hill under the direction of A. C. Mewborn.

Copyright (C) 1971, American Mathematical Society 
We need to show for every $R$-module $M$ that $\mathscr{D}(M)$ is indeed a module over $Q$ in a natural way. Suppose $D$ is a dense right ideal of $R$ and $f: D \rightarrow M$ is an $R$-map. We will denote by $[f]$ the equivalence class of $f$ in $\mathscr{D}(M)$. For $q \in Q$ define an $R$-map $g: q^{-1} D \rightarrow M$ by $g(t)=f(q t)$ for $t \in q^{-1} D$. Then we will define $[f] \cdot q=[g]$. If $f\left(D^{\prime}\right)=(0)$ for some dense right ideal $D^{\prime}$ of $R, g\left(q^{-1} D \cap q^{-1} D^{\prime}\right)=(0)$; i.e., $[g]$ $=[0]$. Hence, this module multiplication is well-defined.

We will call an $R$-module $M$ torsion if $\operatorname{Hom}_{R}\left(M, E\left(R_{R}\right)\right)=(0)$. This particular torsion theory was introduced by Gentile [5] and Jans [6]. The following proposition shows that the filter of right ideals associated with this theory is precisely the filter of dense right ideals.

Proposition 2.1. The following statements are equivalent for the R-module $M$ :

(1) $M$ is torsion.

(2) $\operatorname{Hom}_{R}(N, R)=(0)$ for every $N \subseteq M$.

(3) For $m \in M$, there is a dense right ideal $D$ of $R$ such that $m D=(0)$.

Proof. $(1) \Leftrightarrow(2)$ is noted in Jans [6].

(1) $\Rightarrow(3)$. Suppose for some $m \in M, r(m)$ is not dense, where $r(m)$ is the right annihilator of $m$ in $R$. Then $x \cdot r(m)=(0)$ for some $0 \neq x \in E\left(R_{R}\right)$. Hence, $\psi: m R \rightarrow x R$ given by $\psi(m)=x$ is well-defined. $\psi$ then extends to a nonzero map $M \rightarrow E\left(R_{R}\right)$, a contradiction.

(3) $\Rightarrow(1)$. Suppose $\operatorname{Hom}_{R}\left(M, E\left(R_{R}\right)\right) \neq(0)$. Then there is $f \in \operatorname{Hom}_{R}\left(M, E\left(R_{R}\right)\right)$ and $m \in M$ with $f(m) \neq 0 . m D=(0)$ for some dense right ideal $D$ of $R$; hence, $f(m) D=(0)$, a contradiction.

Hence, for any $R$-module $M$ we have a torsion submodule $M_{0}=\{m \in M \mid m D=(0)$ for some dense right ideal $D$ of $R$. If $M_{0}=(0), M$ is called torsion free.

LeMmA 2.2. If $D$ is a dense right ideal of $R, D Q$ is a dense right ideal of $Q$. If $D_{\alpha}$ is a dense right ideal of $Q, D_{\alpha} \cap R$ is a dense right ideal of $R$.

Proof. Recall $E\left(Q_{Q}\right) \simeq\left(E\left(R_{R}\right)\right)_{Q}$. Hence if $x D Q=(0)$ for some $x \in E\left(Q_{Q}\right), x=0$ since $x D=(0)$.

Suppose $y\left(D_{\alpha} \cap R\right)=(0)$ for some $0 \neq y \in E\left(R_{R}\right)$. For some $a \in R, 0 \neq y a \in R$. Then $D^{\prime}=\left\{q \in Q \mid a q \in D_{\alpha}\right\}$ is $Q$-dense. Thus, $y a q \neq 0$ for some $q \in D^{\prime}$. But $y a q\left(q^{-1} R\right)=(0)$, a contradiction.

Proposition 2.3. A $Q$-module $M$ is torsion free over $Q$ if and only if it is torsion free over $R$.

Proof. Let $m \in M$ and suppose $m D=(0)$ for some dense right ideal $D$ of $R$. Then $m D Q=(0)$. Thus, if $M$ is torsion free over $Q, M$ is torsion free over $R$. Next, suppose $m D_{\alpha}=(0)$ for some dense right ideal $D_{\alpha}$ of $Q$. Then $m\left(D_{\alpha} \cap R\right)=(0)$. Hence, if $M$ is torsion free over $R, M$ is torsion free over $Q$.

In particular, we have shown $\mathscr{D}(M)$ is torsion free over $R$ and $Q$. 
If $A$ and $B$ are $R$-modules and $A \subseteq B$, then we say $A$ is rationally closed in $B$ if for every $b \in B, B \notin A, b^{-1} A$ is not dense. We will denote by $\mathrm{Cl}(A)$ the rational closure of $A$ in $E(A)$; i.e., $\mathrm{Cl}(A)=\left\{x \in E(A) \mid x^{-1} A\right.$ is dense $\}$.

LEMMA 2.4 (GABRIEL). If $D$ is a dense right ideal of $R$ and $H$ is any right ideal of $R$ such that $d^{-1} H$ is dense for every $d \in D$, then $H$ is dense.

Proof. Suppose $x H=(0)$ for some $x \in E\left(R_{R}\right)$. Then $x d\left(d^{-1} H\right)=(0)$ for every $d \in D$. Hence, $x d=0$ for every $d \in D$. Then $x=0$ since $D$ is dense.

Proposition 2.5. Let $M$ be an R-module. Then the torsion submodule of $M, M_{0}$, is rationally closed in $M$.

Proof. Let $m \in M$ and suppose $m^{-1} M_{0}$ is dense. Then for every $x \in m^{-1} M_{0}$, there is a dense right ideal $D^{x}$ of $R$ such that $(m x) D^{x}=(0)$. Let $A=\sum_{x \in D} x D^{x}$. Then $m A=(0)$. If $y \in m^{-1} M_{0}, y^{-1} A \supseteq D^{x}$ and so is dense. Thus, $A$ is dense by Lemma 2.4 ; i.e., $m \in M_{0}$.

It follows immediately that $M / M_{0}$ is torsion free and $(\mathrm{Cl}(M))_{0}=\mathrm{Cl}\left(M_{0}\right)$. In the case where $M$ is already torsion free we have the following relationship between $M$ and $\mathscr{D}(M)$.

Proposition 2.6. The following statements are equivalent for an $R$-module $M$ :

(1) $M$ is torsion free.

(2) The natural $R$-map $\phi: M \rightarrow \mathscr{D}(M)$ is an embedding.

(3) $\mathscr{D}(M)$ is isomorphic to $\mathrm{Cl}(M)$, the rational closure of $M$ in $E(M)$, as an $R$ module.

Proof. (1) $\Rightarrow(2) . \phi$ is given by $\phi(m)=\left[f_{m}\right]$ for $m \in M$, where $f_{m}(x)=m x, x \in R$. If $\phi(m)=[0]$ for some $m \in M, m D=(0)$ for some dense right ideal $D$ of $R$; hence $m=0$.

(2) $\Rightarrow$ (3). Let $x \in \mathrm{Cl}(M)$; i.e., $x^{-1} M$ is dense. Then $f_{x}: x^{-1} M \rightarrow M$ given by $f_{x}(y)=x y$ for $y \in x^{-1} M$ is well-defined. Suppose for some dense right ideal $D$ of $R$, $f_{x}\left(x^{-1} M \cap D\right)=(0)$. Let $D^{\prime}=x^{-1} M \cap D$. Now $y^{-1} D^{\prime}$ is dense for every $y \in x^{-1} M$ and $f_{x}(y) y^{-1} D^{\prime}=(0)$. Then $\left[f_{x}\right] \cdot y=\phi(x y)=(0)$. But $x y \neq 0$ for some $y \in x^{-1} M$, a contradiction. Thus, $f_{x}\left(D^{\prime}\right) \neq(0)$ for every dense right ideal $D$; i.e., $\left[f_{x}\right] \neq[0]$. Then the mapping $\phi_{1}: \mathrm{Cl}(M) \rightarrow \mathscr{D}(M)$ given by $\phi_{1}(x)=\left[f_{x}\right]$ is an imbedding. If $[f] \in \mathscr{D}(M)$, say $f: D \rightarrow M$ where $D$ is some dense right ideal of $R$, then $f(t)=z t$ for some $z \in E(M)$, and every $t \in D$. Since $z D \subseteq M, z \in \mathrm{Cl}(M)$; thus, $\phi_{1}(z)=[f]$. Therefore, $\phi_{1}$ is an isomorphism.

(3) $\Rightarrow(1) . \mathscr{D}(M)$ is torsion free over $R$; hence, $\mathrm{Cl}(M)$ and $M$ are torsion free.

THEOREM 2.7. Let $M$ be an $R$-module. Then $\mathscr{D}(M)$ is isomorphic to $\mathrm{Cl}(M) / \mathrm{Cl}\left(M_{0}\right)$ and $\mathrm{Cl}(\mathscr{D}(M))$ is isomorphic to $\mathscr{D}\left(M / M_{0}\right)$.

Proof. Suppose $x+\mathrm{Cl}\left(M_{0}\right) \in \mathrm{Cl}(M) / \mathrm{Cl}\left(M_{0}\right)$. Then $x^{-1} M$ is dense and we can define $f_{x}: x^{-1} M \rightarrow M$ as before. Define $\psi: \mathrm{Cl}(M) / \mathrm{Cl}\left(M_{0}\right) \rightarrow \mathscr{D}(M)$ by 
$\psi\left(x+\mathrm{Cl}\left(M_{0}\right)\right)=\left[f_{x}\right] . \psi$ is well-defined since if $x \in \mathrm{Cl}\left(M_{0}\right), f_{x}$ is zero on some dense right ideal of $R$. Ker $\psi$ is clearly zero. If $D$ is a dense right ideal of $R$ and $g: D \rightarrow M$ is an $R$-map, $g(t)=y t$ for some $y \in \mathrm{Cl}(M)$ and all $t \in D$. Then $\psi\left(y+\mathrm{Cl}\left(M_{0}\right)\right)=[g]$; hence, $\psi$ is an isomorphism.

Let $\pi: M \rightarrow M / M_{0}$ be the natural map. If $f \in \operatorname{Hom}_{R}(D, M)$ for $D$ a dense right ideal of $R, \pi \circ f \in \operatorname{Hom}_{R}\left(D, M / M_{0}\right)$. Hence, define $\eta: \mathscr{D}(M) \rightarrow \mathscr{D}\left(M / M_{0}\right)$ by $\eta([f])=[\pi \circ f]$ for every $[f] \in \mathscr{D}(M) . \eta$ is clearly well-defined. Suppose $f(D) \subseteq M_{0}$; i.e., $\pi \circ f=0$. Then for $t \in D$, there is some $x \in \mathrm{Cl}\left(M_{0}\right)$ such that $f(t)=x t$. $x D^{\prime}=(0)$ for some dense right ideal $D^{\prime}$ of $R$; hence, $f\left(D \cap D^{\prime}\right)=(0)$. Thus, $[f]=[0]$; i.e., $\eta$ is an imbedding. Then if the image of $\eta$ is large in $\mathscr{D}\left(M / M_{0}\right)$ we will be done, since $M / M_{0}$ is torsion free and hence $\mathscr{D}\left(M / M_{0}\right)$ is rationally closed by Proposition 2.6. Let $[0] \neq[g] \in \mathscr{D}\left(M / M_{0}\right)$ where $g: D \rightarrow M / M_{0}, D$ some dense right ideal of $R$. There is $z \in \mathrm{Cl}\left(M / M_{0}\right)$ such that $g(t)=z t$ for every $t \in D$. Then $0 \neq z a \in M / M_{0}$ for some $a \in R$. Choose $m \in z a$ and define $g_{1}: a^{-1} D \rightarrow M$ by $g_{1}(t)=m t$ for $t \in a^{-1} D$. Then $\pi \circ g_{1}(t)=z a t$ for $t \in a^{-1} D$. Hence, $\eta\left(\left[g_{1}\right]\right)=[g] \cdot a$.

3. Suppose $M$ and $N$ are $R$-modules and $f: M \rightarrow N$ is an $R$-map. Then the map $\mathscr{D}(f): \mathscr{D}(M) \rightarrow \mathscr{D}(N)$ given by $\mathscr{D}(f)([g])=[f \circ g]$ for $[g] \in \mathscr{D}(M)$ is well-defined and is a $Q$-map. Hence, $\mathscr{D}$ is a covariant functor from the category of $R$-modules to the category of $Q$-modules. $\mathscr{D}$ is left exact since $\operatorname{Hom}_{R}(D, \cdot)$ is left exact for $R$-modules $D . \mathscr{D}$ is not right exact in general. For example, let $B=\left\{x_{\alpha} \mid \alpha \in[0,1]\right\}$ be a basis for a vector space over $Z_{2}$ and give $B$ the associative multiplication

$$
\begin{aligned}
x_{\alpha} \cdot x_{\beta} & =x_{\alpha+\beta} & & \text { if } \alpha+\beta \leqq 1, \\
& =0 & & \text { if } \alpha+\beta>1 .
\end{aligned}
$$

Then the induced ring $R$ has a unique minimal dense right ideal $D$ generated by $\left\{x_{\alpha} \mid \alpha>0\right\}$. It can be shown $D$ is not projective; hence, for some exact sequence $0 \rightarrow A \rightarrow B \rightarrow C \rightarrow 0$ of $R$-modules, $0 \rightarrow \operatorname{Hom}_{R}(D, A) \rightarrow \operatorname{Hom}_{R}(D, B) \rightarrow$ $\operatorname{Hom}_{R}(D, C) \rightarrow 0$ is not exact. In this case, for any $R$-module $M, \mathscr{D}(M) \simeq$ $\operatorname{Hom}_{R}(D, M)$; i.e., $\mathscr{D}$ is not exact.

In contrast, the functor $\mathscr{T}$ from the category of $R$-modules to the category of $Q$-modules which associates an $R$-module $M$ with $\mathscr{T}(M)=M \otimes_{R} Q$ (the usual extension of scalars) is right exact. $\mathscr{T}$ is exact if and only if ${ }_{R} Q$ is flat.

Proposition 3.1. $\mathscr{D}$ is exact if every dense right ideal is projective.

Proof. Suppose $0 \rightarrow A \rightarrow B \stackrel{\pi}{\rightarrow} C \rightarrow 0$ is exact. We wish to show $\mathscr{D}(\pi)$ is an epimorphism. If $D$ is a dense right ideal of $R$ and $f: D \rightarrow C$, then there is $g: D \rightarrow B$ such that $\pi \circ g=f$, since $D$ is projective. $\mathscr{D}(\pi)([g])=[f]$ by definition of $\mathscr{D}(\pi)$.

It is evident that if $R$ contains a minimal dense right ideal $D, \mathscr{D}$ is exact if and only if $D$ is projective. There exist such rings which also have nonprojective dense right ideals, for example an infinite product of fields. Hence, the converse of Proposition 3.1 is not true.

The proof of the following well-known lemma is omitted. 
Lemma 3.2 (TEWARI). If either $M$ or $N$ is a finitely generated and projective $R$-module, then $N \otimes_{R} \operatorname{Hom}_{R}(M, R)$ is naturally isomorphic to $\operatorname{Hom}_{R}(M, N)$.

Proposition 3.3. If $M$ is a finitely generated and projective $R$-module, then $\mathrm{Cl}(M)$ is isomorphic to $\mathscr{D}(M)$ and $\mathscr{D}(M)$ is isomorphic to $\mathscr{T}(M)$.

Proof. Taking direct limits over the collection of dense right ideals of $R$ we have $\mathscr{T}(M)=M \otimes_{R} Q \simeq M \otimes_{R} \operatorname{inj} \lim \operatorname{Hom}_{R}(D, R) \simeq \operatorname{inj} \lim M \otimes_{R} \operatorname{Hom}_{R}(D, R)$ since the tensor product commutes with direct limits. Then by Lemma 3.2 we have

$$
\mathscr{T}(M) \simeq \operatorname{inj} \lim \operatorname{Hom}_{R}(D, M)=\mathscr{D}(M)
$$

Since $M$ is projective, $M_{0}=(0)$ and the proposition follows.

REMARK. If every dense right ideal of $R$ is finitely generated and projective, then by the proof of this proposition we have $\mathscr{D}(M) \simeq \mathscr{T}(M)$. It is not difficult to show that the isomorphisms of Proposition 3.3 need not hold in general.

Proposition 3.4. Let $\left\{M_{i}\right\}_{1}^{n}$ be a collection of $R$-modules. Then $\mathscr{D}\left(\prod_{1}^{n} M_{i}\right)$ is isomorphic to $\prod_{1}^{n} \mathscr{D}\left(M_{i}\right)$; i.e., $\mathscr{D}$ commutes with finite products.

Proof. Define $\psi: \mathscr{D}\left(\prod_{1}^{n} M_{i}\right) \rightarrow \prod_{1}^{n} \mathscr{D}\left(M_{i}\right)$ by $\psi([f])=\left(\left[\pi_{1} \circ f\right],\left[\pi_{2} \circ f\right], \ldots,\left[\pi_{n} \circ f\right]\right)$ where $\pi_{k}$ is the $k$ th projection for each $k$, and $[f] \in \mathscr{D}\left(\prod_{1}^{n} M_{i}\right)$. Ker $\psi=(0)$ clearly. It is straightforward to show $\psi$ is an epimorphism since the intersection of a finite number of dense right ideals is again dense.

It is not true in general that $\mathscr{D}$ commutes with arbitrary products. We can say the following:

Proposition 3.5. $\mathscr{D}$ commutes with arbitrary direct products if and only if $R$ contains a minimal dense right ideal.

Proof. If $R$ contains a minimal dense right ideal $D, \mathscr{D}(M) \simeq \operatorname{Hom}_{R}(D, M)$ for every $R$-module $M$. Then if $\left\{M_{\alpha}\right\}_{\alpha \in I}$ is a collection of $R$-modules,

$$
\mathscr{D}\left(\prod_{\alpha \in I} M_{\alpha}\right) \simeq \operatorname{Hom}_{R}\left(D, \prod_{\alpha \in I} M_{\alpha}\right) \simeq \prod_{\alpha \in I} \operatorname{Hom}_{R}\left(D, M_{\alpha}\right) \simeq \prod_{\alpha \in I} \mathscr{D}\left(M_{\alpha}\right) .
$$

Conversely, consider $\left\{D_{\alpha} \mid D_{\alpha}\right.$ is a dense right ideal of $\left.R\right\}$. Let $M=\prod_{\alpha \in I} R / D_{\alpha}$. Then $\mathscr{D}(M) \simeq \prod_{\alpha \in I} \mathscr{D}\left(R / D_{\alpha}\right)=(0)$. Hence, $M$ is torsion. Then if we choose $m \in M$ such that $\pi_{\alpha}(m)=1+D_{\alpha}$ for each $\alpha, m D=(0)$ for some dense right ideal $D$. But then $D \subseteq D_{\alpha}$ for each $\alpha$.

On the same note we observe the following:

Proposition 3.6. The product of torsion modules is again torsion if and only if $R$ contains a minimal dense right ideal.

Proof. If $D$ is a minimal dense right ideal of $R$ and $\left\{M_{\alpha}\right\}_{\alpha \in I}$ is a collection of torsion modules, then $M_{\alpha} D=(0)$ for each $\alpha$; hence, $\left(\prod_{\alpha \in I} M_{\alpha}\right) D=(0)$. Thus, $\prod_{\alpha \in I} M_{\alpha}$ is torsion. 
Conversely, if the product of torsion modules is torsion, let $M=\prod_{\alpha \in I} R / D_{\alpha}$ where $I$ runs over the collection of dense right ideals of $R$. $M$ is torsion; hence, $R$ contains a minimal dense right ideal by the argument used in Proposition 3.5.

A ring $R$ is said to be left perfect (see Bass [1]) if $R$ has d. c. c. on principal right ideals.

Proposition 3.7. If $R$ is left perfect, then $R$ contains a minimal dense right ideal.

Proof. Let $\left\{D_{\alpha}\right\}_{\alpha \in I}$ be the collection of dense right ideals of $R$. Then $\bigcap_{\alpha \in I} D_{\alpha}$ will be dense if we can show that $x\left(\bigcap_{\alpha \in I} D_{\alpha}\right) \neq(0)$ for $0 \neq x \in E\left(R_{R}\right)$. For $\alpha_{1} \in I$, $x d_{1} \neq 0$ for some $d_{1} \in D_{\alpha_{1}}$. Suppose $d_{1} R \nsubseteq D_{\alpha_{2}}$ for some $\alpha_{2} \in I$. Then there is $d_{2} \in\left(D_{\alpha_{1}} \cap D_{\alpha_{2}}\right) \cap d_{1}^{-1}\left(D_{\alpha_{1}} \cap D_{\alpha_{2}}\right)$ such that $\left(x d_{1}\right) d_{2} \neq 0$ and $d_{1} R \supsetneqq d_{1} d_{2} R$. By this process we get a proper descending chain $d_{1} R \supsetneq d_{1} d_{2} R \supsetneq \cdots$ with $x d_{1} d_{2} \cdots d_{n} \neq 0$ for every $n$. Since $R$ is left perfect, it must be the case that, for some $n, d_{1} d_{2} \cdots d_{n} R$ $\subseteq D_{\alpha}$ for every $\alpha \in I$.

COROLlaRY 3.8 (JANS [6]). If $R$ is left perfect, then the production of torsion modules is again torsion.

4. Let $M$ be an $R$-module and $H=\operatorname{Hom}_{R}(E(M), E(M))$. The rational completion $M^{*}$ of $M$ is defined to be

$$
\{x \in E(M) \mid \text { if } f \in H, f(M)=(0), \text { then } f(x)=0\} .
$$

$M$ is called faithful if for each $0 \neq x \in R$ there exists $m \in M$ such that $m x \neq 0$.

THEOREM 4.1. If $M$ is a torsion free and faithful $R$-module then $\mathscr{D}(M) \simeq \mathrm{Cl}(M)$ $=M^{*}$.

Proof. $\mathscr{D}(M) \simeq \mathrm{Cl}(M)$ by Proposition 2.6. If $x \in \mathrm{Cl}(M)$ and $f: E(M) \rightarrow E(M)$ has the property that $f(M)=(0)$, then $f(x) x^{-1} M=(0)$; i.e., $f(x)=0$. Hence, $x \in M^{*}$.

Suppose next that $x \in M^{*}$ and $K=x^{-1} M$ is not dense; i.e., $z K=(0)$ for some $0 \neq z \in E\left(R_{R}\right)$. For some $a \in R, 0 \neq z a \in R$. Then $m(z a) \neq 0$ for some $m \in M$ since $M$ is faithful. The map $\alpha: K+a R \rightarrow M$ given by $\alpha(k+a t)=m(z a) t$, for $k \in K$ and $t \in R$, is well-defined since $z a\left(a^{-1} K\right)=(0)$. Furthermore, $\alpha$ is nonzero. Hence, $\alpha(t)$ $=y t$ for some $0 \neq y \in E(M)$ and every $t \in K+a R$. $\alpha(K)=(0)$ so that $y K=(0)$. But then $f: M+x R \rightarrow M$ given by $f\left(m_{1}+x t\right)=y t$ for $m_{1} \in M, t \in R$, is well-defined. $f(M)=(0)$ and $f(x)=y \neq 0$, a contradiction.

Examples exist in the literature which show the hypothesis that $M$ is torsion free is not sufficient for the above theorem (e.g., Osofsky [10]).

If $M$ is an $R$-module, $\operatorname{Hom}_{R}(Q, M)$ becomes a $Q$-module by defining $f \cdot q(p)$ $=f(q p)$ for $f \in \operatorname{Hom}_{R}(Q, M)$ and $p, q \in Q$. The following lemma may be found in Cartan-Eilenberg [2] in a more general form.

Lemma 4.2. If $M$ is an injective $R$-module, then $\operatorname{Hom}_{R}(Q, M)$ is an injective Q-module. 
Proof. Let $0 \rightarrow A \rightarrow B$ be an exact sequence of $Q$-modules and let $f: A \rightarrow \operatorname{Hom}_{R}(Q, M)$ be a $Q$-map. Define $\psi: A \rightarrow M$ by $\psi(a)=f(a)(1)$ for $a \in A$. Since $M$ is $R$-injective and $\psi$ is an $R$-map, there is an $R$-map $f_{1}: B \rightarrow M$ extending $\psi$. Define $g: B \rightarrow \operatorname{Hom}_{R}(Q, M)$ by $g(b)(q)=f_{1}(b q)$ for $b \in B, q \in Q$. Then $g$ is a $Q$-map extending $f$.

Lemma 4.3. If $M$ is a torsion free $Q$-module, $M$ is isomorphic to $\operatorname{Hom}_{R}(Q, M)$.

Proof. Let $f \in \operatorname{Hom}_{R}(Q, M)$. Then for $q \in Q,(f(q)-f(1) q) q^{-1} R=(0)$; hence, $f(q)=f(1) q$ since $M$ is torsion free. Thus, $\operatorname{Hom}_{R}(Q, M)=\operatorname{Hom}_{Q}(Q, M) \simeq M$.

THEOREM 4.4. A torsion free $Q$-module is $R$-injective if and only if it is $Q$-injective.

Proof. Let $M$ be a torsion free $Q$-module. If $M$ is $R$-injective, $M$ is $Q$-injective by Lemmas 2.2 and 2.3 .

Conversely, suppose $M$ is $Q$-injective. Let $H$ be a right ideal of $R$ and let $f: H \rightarrow M$ be an $R$-map. Then $0 \rightarrow \mathscr{D}(H) \rightarrow \mathscr{D}\left(R_{R}\right) \simeq Q$ is an exact sequence of $Q$-modules and $\mathscr{D}(f): \mathscr{D}(H) \rightarrow \mathscr{D}(M)$ is a $Q$-map. $\mathscr{D}(M) \simeq \mathrm{Cl}(M)=M$. Hence, $\mathscr{D}(f)$ lifts to a $Q$-map $\psi: \mathscr{D}(R) \rightarrow \mathscr{D}(M)$. Since $H$ and $R$ are torsion free, we may assume $H \subseteq \mathscr{D}(H)$ and $R \subseteq \mathscr{D}(R)$. Then $\mathscr{D}(f)$ restricted to $H$ is $f$ and $\psi$ restricted to $R$ is an $R$-map extending $f$. Hence, $M$ is $R$-injective by Baer's criterion.

\section{REFERENCES}

1. H. Bass, Finitistic dimension and a homological generalization of semi-primary rings, Trans. Amer. Math. Soc. 95 (1960), 466-488. MR 28 \#1212.

2. H. Cartan and S. Eilenberg, Homological algebra, Princeton Univ. Press, Princeton, N. J., 1956. MR 17, 1040.

3. S. E. Dickson, A torsion theory for Abelian categories, Trans. Amer. Math. Soc. 121 (1966), 223-235. MR 33 \#162.

4. B. Eckmann and A. Schopf, Über injektive Moduln, Arch. Math. 4(1953), 75-78. MR 15, 5.

5. E. Gentile, Singular submodule and injective hull, Nederl. Akad. Wetensch. Proc. Ser. A65 = Indag. Math. 24 (1962), 426-433. MR 26 \#2478.

6. J. P. Jans, Some aspects of torsion, Pacific J. Math. 15 (1965), 1249-1259. MR 33 \#163.

7. R. E. Johnson, The extended centralizer of a ring over a module, Proc. Amer. Math. Soc. 2 (1951), 891-895. MR 13, 618.

8. J. Lambek, Lectures on rings and modules, Blaisdell, Waltham, Mass., 1966. MR 34 \#5857.

9. A. C. Mewborn, Generalized centralizers of modules, Duke Math. J. 35 (1968), 575-580.

10. B. L. Osofsky, A generalization of quasi-Frobenius rings, J. Algebra 4 (1966), 373-387. MR 34 \#4305.

University of North Carolina,

Chapel Hill, North Carolina 27514

University OF South Carolina,

Columbia, South Carolina 29208 